\title{
Las salas de redacción en la era digital, la percepción y los principales retos de la comunidad virtual de periodistas y colaboradores, caso La Barra Espaciadora, Ecuador
}

\author{
The newsrooms in the digital age, the perception and \\ the main challenges of the virtual community of \\ journalists and collaborators, the case of La Barra \\ Espaciadora, Ecuador
}
As redações na era digital, a percepção e os principais desafios da comunidade virtual de jornalistas e colaboradores, o caso de La Barra Espaciadora, Equador

\author{
Yalilé Loaiza Ruiz ${ }^{1}$ \\ Universidad de Los Hemisferios (Ecuador) \\ yalilel@uhemisferios.edu.ec
}

Fecha de recepción: 06 de febrero de 2018

Fecha de recepción evaluador: 30 de septiembre de 2018

Fecha de recepción corrección: 14 de noviembre de 2018

\footnotetext{
${ }^{1}$ Yalilé Loaiza Ruiz es Maestranda en investigación de la Comunicación Digital por la Universidad de Los Hemisferios. Desempeñó el cargo de Coordinadora Académica y Docente en la Facultad de Comunicación de la Universidad de Los Hemisferios. Es Comunicadora Social con un minor en Producción Cinematográfica. Fue parte del equipo de la revista digital La Barra Espaciadora como Coordinadora de Narrativas Web y Redes Sociales. Fue también Coordinadora de edición en el Sello Editorial Razón y Palabra. Posee cinco años en medios de comunicación tradicionales y digitales. Ha participado en la producción de varios cortometrajes y como dialoguista en series de televisión. Además, ha publicado artículos en revistas indexadas. https://orcid.org/0000-0002-7277-7084
} 


\title{
Resumen
}

En la presente investigación, se plantea un primer esbozo para describir cómo funcionan las salas de redacción en la era digital y observar cómo interactúan y cuáles son los principales retos de los miembros de la comunidad virtual de colaboradores y periodistas del medio digital ecuatoriano La Barra Espaciadora. Se observa cómo es percibida la estructura del medio por la comunidad, así como las falencias en cuando al modelo de negocio y distribución del tiempo y funciones.

Palabras claves: salas de redacción digitales, comunidades virtuales, medios digitales, periodismo.

\begin{abstract}
In the following research, a first outline is presented to describe how newsrooms function in the digital age and observe how they interact and what are the main challenges of the members of the virtual community of collaborators and journalists of the Ecuadorian digital media La Barra Espaciadora. It is observed how the structure of the environment is perceived by the community, as well as the shortcomings in the business model and distribution of time and functions.
\end{abstract}

Keywords: digital newsrooms, virtual communities, digital media, journalism.

\section{Resumo}

Na pesquisa a seguir, um primeiro esboço é apresentado para descrever como as redações funcionam na era digital e observar como elas interagem e quais são os principais desafios dos membros da comunidade virtual de colaboradores e jornalistas da mídia digital equatoriana La Barra Espaciadora. Observa-se como a estrutura do ambiente é percebida pela comunidade, bem como as deficiências no modelo de negócio e distribuição de tempo e funções.

Palavras-chave: redações digitais, comunidades virtuais, mídia digital, jornalismo.

\section{Introducción}

El ciberespacio cambió nuestras vidas en maneras que escasamente podíamos imaginar (Rheingold, 2007, p. 11), desde la forma de relacionarnos con nuestros pares hasta cómo trabajar y adaptarnos al ambiente digital. Además de reconfigurarse nociones básicas como el espacio y el tiempo, internet también cambió a los oficios -o los está cambiando.

Un ejemplo de lo anterior es el trabajo del periodista. Con "la aparición de internet como medio de comunicación se ha dado paso a definir un nuevo perfil profesional (del periodista) caracterizado por el uso de renovadas técnicas como la hipertextualidad, la multimedialidad y la interactividad" (Gonzales, Rodriguez, \& Rivera, 2016, p. 1). Lo que 
demanda una adaptabilidad del periodista tradicional a la nueva plataforma y a los lenguajes que esta requiere a la hora de crear y difundir los contenidos periodísticos.

Con internet y la era digital no solo los medios de comunicación tradicionales buscaron adaptar sus contenidos al ciberespacio, sino que medios nativos digitales aparecieron en el radar. Se empezó entonces a cuestionar si la organización, la forma de trabajo y las prácticas periodísticas tradicionales se adaptarían o cambiarían.

En los medios digitales nativos "al no tener como punto de partida el medio tradicional, sus reporteros trabajan al ritmo de la generación de la información, es decir, establecen sus propias rutinas de trabajo" (Rivera Rogel, Pereira-Fariña, \& Yaguache Quichimbo, 2015, p. 15). No obstante, "las rutinas de producción de información se organizan de acuerdo a las necesidades de cada medio de comunicación" (Rivera Rogel et al., 2015, p. 17).

Pero con internet no solo cambian las rutinas, sino también los contenidos. Tanto medios tradicionales como nativos digitales aún buscan la fórmula de éxito frente a los nuevos lenguajes. Si bien no existe nada definido, salvo que estamos en un momento de transición, se cree que "el lenguaje ciberperiodístico plantea, en fin, una amalgama de códigos que, sin duda, desembocará en el alumbramiento de nuevos géneros y en profundas mutaciones futuras en las formas de elaborar contenidos informativos" (Salaverría, 2009, p. 21). Al momento, la convergencia entre lo tradicional y lo digital se convierte en una de las formas de ejercer el periodismo en la web.

Dicha convergencia abarca a los géneros, rutinas, formas de difusión y funcionalidad en las salas de redacción. Se debe tener presente que "ya existían diferentes formas de convergencia en los medios tradicionales (...) pero es innegable que el nuevo medio (internet) potenció y enriqueció el fenómeno, hasta el punto de que está entre las áreas más estudiadas de la investigación en periodismo" (Canavilhas, 2013, p. 11). De ahí la importancia de analizar cómo afecta el nuevo espacio a los ambientes ya conocidos.

Investigadores como Javier Díaz-Noci (2008) sostienen que un cibermedio debe poseer tres características principales: multimedialidad, interactividad e hipertextualidad. Es decir, que más allá de ser nativos digitales, para ser llamados cibermedios, sus contenidos deben contar con los elementos mencionados.

En Ecuador, existen medios nativos digitales pero su presencia es menor a los tradicionales que poseen versiones digitales. Los medios nativos digitales ecuatorianos, pese a tener como espacio a internet, "no son más multimediales que los de prensa y, a pesar de su origen, son poco hipertextuales" (Aguirre Mayorga, Odriozola Chené, \& Bernal Suárez, 2015, p. 498). No obstante, "los cibermedios nativos digitales están a medio camino en todos los aspectos del ciberperiodismo" (Aguirre Mayorga et al., 2015, p. 498). Este hecho podría darse por la reconfiguración del trabajo en las salas de redacción y los aportes de la comunidad de periodistas y colaboradores del medio digital.

Además, los medios nativos funcionan con base en el trabajo colaborativo. En algunos casos, los colaboradores frecuentes son personas ajenas al periodismo. No obstante, las salas de redacción integradas con periodistas y colaboradores que no, 
necesariamente, comparten un mismo espacio físico, poseen la capacidad de atender a las audiencias multiplataformas y de beneficiar a los medios por su bajo costo (Salaverría, 2010, p. 30).

Es esa integración en el ciberespacio que genera un equipo de trabajo -una comunidad- que puede mantener interacciones distintas a las del equipo de trabajadores de un medio tradicional.

El ambiente elegido para la investigación es el medio nativo digital ecuatoriano La Barra Espaciadora- LBE. Este medio nació, en 2013, por iniciativa de un grupo de periodistas ecuatorianos independientes. Se considera una revista de periodismo narrativo y está conformada por "articulistas, fotógrafos, editores y demás apasionados por el mundo de la comunicación y del periodismo digital, así como por una red satélite de colaboradores externos" (La Barra Espaciadora, s/f-b, párr. 1).

El trabajo del medio es "de carácter colaborativo e independiente que alimenta sus contenidos con aportes voluntarios de sus lectores, sin importar su lugar de origen o de residencia, sean estos periodistas o no" (La Barra Espaciadora, s/f-b, párr. 1). Asimismo, dentro de sus principios establece que "no recibe ni recibirá jamás dinero de ningún personaje en el ejercicio político ni de ninguna organización que represente intereses políticos o de grupos de poder económico de cualquier naturaleza" (La Barra Espaciadora, s/f-a, párr. 3).

La Barra Espaciadora posee una sala redacción digital, es decir, que no se asienta sobre un espacio físico sino en un entorno virtual. La interacción con los colaboradores y periodistas, principalmente, sucede en línea. Inclusive existen personas de su equipo de trabajo que solamente conocen a otras por medio de redes sociales digitales y no cara a cara.

En Ecuador, "la investigación sobre los efectos del ciberperiodismo es un sector que todavía no se ha asentado" (Odriozola-Chéné, 2016, p. 71). Además, se considera que aún los medios digitales se encuentran en la primera fase del ciberperiodismo (Rivera Rogel et al., 2015, p. 221). Según Sembramedia, en el país existen 50 cibermedios (s/f), cada uno de ellos trabaja con su sala de redacción e intenta adaptar a ella los procesos de creación y difusión de contenidos que más les convenga.

Es por lo anterior que se eligió al medio como el ambiente de trabajo de esta investigación, además de su apertura frente al investigador para el libre acceso a los datos del medio.

Con la breve explicación anterior, el presente trabajo se plantea la siguiente pregunta de investigación: ¿Cómo debe funcionar la comunidad virtual de colaboradores y periodistas, sus rutinas periodísticas y el trabajo en la sala de redacción de un medio digital -LBE- para la creación y difusión de contenidos? Asimismo, se define como problema de investigación al siguiente: Las salas de redacción en la era digital generan una nueva relación entre las comunidades virtuales de colaboradores y periodistas a la hora de crear contenidos y difundirlos, además de la adaptación de las rutinas periodísticas tradicionales al ciberespacio. 
Para el presente documento, me referiré principalmente a los hallazgos relacionados con los siguientes objetivos: a) Describir la estructura y modo de trabajo en la sala de redacción digital de LBE; y b) Observar la comunicación, trabajo colectivo y principales desafíos de la comunidad de colaboradores y periodistas en la sala de redacción digital de $\mathrm{LBE}$.

\section{Marco teórico}

\section{Comunidades virtuales}

Se puede entender a las comunidades virtuales como la organización de usuarios en internet que comparten intereses, gustos, preferencias y puntos de vista similares, que se reúnen en el espacio virtual para compartir información, trabajar o comunicarse con sus pares. Es allí donde se crean nuevas formas de conocimiento o donde "la nueva cultura del conocimiento se aviva con las múltiples modalidades del saber" (Jenkins, 2015, p. 168). Además, se generan interacciones cuya base es la cooperatividad. Rheingold (2007) describía lo anterior así:

Las multitudes inteligentes consisten en personas que actúan concertadamente inclusive si no se conocen. Las personas que forman parte de estas multitudes cooperan entre sí de formas que antes no eran posibles porque ahora llevan dispositivos que poseen ambas: la comunicación y las capacidades computacionales (p. 12).

Asimismo, el encuentro entre pares lleva a "la construcción de una identidad colectiva" (Recuero, Amaral, \& Monteiro, 2012, p. 3). Esta construcción colectiva se produce por la homofilia, considerada como "la tendencia de las personas que son similares a conectarse entre sí" (Recuero et al., 2012, p. 3), si a lo anterior le sumamos el sin fronteras, una característica atribuida al internet, podríamos decir que un factor que favorece a la creación de identidad colectiva es que se crean "zonas de contacto temporales entre culturas geográficamente dispersas" (Jenkins, 2015, p. 185). Esto permite que personas dispersas alrededor del mundo puedan conectarse entre sí.

En el soporte online conviven dos clases de periodistas, el tradicional, que produce noticias para los medios tradicionales (prensa, radio y Tv), que a su vez forman parte de las versiones digitales de estos mismos medios, y un periodista digital, nacido en Internet con experiencia en redacción ciberperiodística, y que es quien impulsa este proceso de cambio en todo el mundo (Gonzales et al., 2016, p. 1).

Es dentro de esta convergencia de periodistas, métodos y audiencias donde también se evidencia la unión de varios profesionales del oficio que buscan aprender y descubrir cómo manejar sus contenidos en internet, mediante grupos cuyo nido es una red social - Facebook for journalists puede ser un ejemplo de lo dicho.

No obstante, la convergencia y participación de la audiencia son factores que los medios de comunicación todavía no saben cómo manejar. Existen dificultades para encajar a esos nuevos actores a las estructuras existentes. Es así como, la publicación multimedia online, está lejos de ser consolidada en cuanto a géneros y rutinas de producción periodística (Schmitz Weiss \& Domingo, 2010, pp. 1157-1158). 
La convergencia de los usuarios dentro de la virtualidad genera esas zonas de contacto temporales dispersas de las que Jenkins hablaba. Es así que, tomando las categorías de Manuel Castells, clasificaremos a la realidad en dos: la presencial y la virtual (Fernández Serrano, 2014). Esto para poder referirnos a las resignificaciones del espacio en la realidad virtual ya que "Internet y las tecnologías de la comunicación han cambiado por completo las nociones de espacio y de tiempo en una suerte de transformación definitiva y final de la evolución" (Llorca-Abad \& Cano-Orón, 2016, p. 221) pues "los límites físicos que imponen el espacio y el tiempo parecen difuminarse con la tecnología" (Llorca-Abad \& Cano-Orón, 2016, p. 228).

En el caso del espacio, la reconfiguración en lo borderless o 'sin fronteras'. Ya no se necesita estar en el mismo espacio físico para interactuar con otro. Sin embargo, la interacción se produce dentro de los territorios de internet -una red social, por ejemplo. La nueva noción de espacio se traslada a internet como tal y a los sitios web que este contiene.

Los periodistas en internet encontraron, como otros usuarios, un espacio que les permite conseguir y transmitir información, al mismo tiempo que podían mantenerse al tanto de las actualizaciones de sus colegas y de las figuras públicas.

Ha sido dentro las redes sociales donde se ha creado comunidades virtuales de periodistas, especialmente en Twitter, donde la interacción entre periodistas, periodistasaudiencia y periodistas- fuente es notoria.

Twitter ha pasado de ser una plataforma llena de "información inútil”, a ser una herramienta diaria para un gran porcentaje de periodistas. Quienes la usan para seguir a las fuentes de una historia, para crear marcas personales, para generar mayor transparencia y responsabilidad en su trabajo, y como herramienta de investigación (Canter, 2015, pp. 888-889).

Estas nuevas comunidades desarrolladas en la red permiten a los periodistas "mantenerse en contacto con sus colegas y su red profesional" (Paulussen \& Harder, 2014, p. 2). Así, "encuentran nuevos contactos, y discuten noticias y problemas" (Paulussen \& Harder, 2014, p. 2) de manera similar a como se lo solía hacer en salas de redacción. Por su parte, Hermida manifiesta que las redes sociales permiten al periodista "monitorear la constante transmisión de noticias e información y detectar tendencias y cuestiones bajo el radar de noticias" (2010, p. 302).

Las redes sociales también abrieron el camino para la participación de los usuarios en el periodismo. El periodismo participativo se puede definir como el "proceso de audiencias participando junto a periodistas y entre ellos en la creación de noticias y la construcción de comunidades en torno al periodismo" (Lewis, Holton, \& Coddington, 2014, p. 231). 


\section{Salas de redacción: lo tradicional vs. lo digital}

No es sorpresa que "el advenimiento de las tecnologías digitales ha tenido un profundo efecto disruptivo en los sistemas y procesos tradicionales del periodismo, a los que los medios estaban acostumbrados hasta finales del siglo XX" (Salaverría, 2009, p. 1). De forma tal que no solo las rutinas periodísticas han evolucionado sino también la sala de redacción.

Algunos medios de comunicación nativos digitales notaron que "mediante la creación y gestión de una sala de redacción virtual, la gestión de los medios de comunicación puede reducir drásticamente el coste reduciendo o incluso eliminando la sala de noticias central y física" (Pavlik, 2013, p. 187). Junto a la reducción de costos y a la posibilidad de no necesitar un espacio físico para distribuir el trabajo, las tecnologías digitales trajeron otros beneficios:

Además de multiplicar el abanico de fuentes, han aligerado ciertas labores mecánicas que antaño lastraban su quehacer informativo cotidiano. Las tecnologías digitales, y especialmente internet, han traído consigo un enriquecimiento en los lenguajes informativos, en las modalidades de relación con el público, en la ubicuidad y temporalidad de la información y, en fin, en múltiples ámbitos cruciales de la actividad periodística (Salaverría, 2009, p. 2).

Si bien los beneficios parecieran ser el 'plato fuerte' de la era digital, las nuevas tecnologías también generaron que "los modelos de negocio tradicionalmente empleados por las empresas periodísticas hayan entrado en una profunda crisis" (Salaverría, 2009, p. 2), puesto que con la información accesible para todos, la venta de información no resulta como un buen negocio.

Por otra parte, Ramón Salaverría indica que las salas de redacción de la era digital poseen dos polivalencias: la funcional y la temática. La primera consiste en que el periodista deba realizar más de una tarea dentro de la sala de redacción. La segunda, en cambio, se refiere a la falta de especialización de los periodistas, quienes deben informar sobre variados temas por falta de especialistas, algo que es reservado para salas de redacción bien nutridas (Salaverría, 2009, p. 10).

La convergencia o el uso de salas de redacción digitales es parte de los procesos de innovación periodística. John Pavlik (2013) señala que:

La innovación en los medios de comunicación, que incluye tanto a los periodistas profesionales como a los periodistas ciudadanos que generan contenido de noticias, se encuentra al menos en cuatro dimensiones: (1) crear, entregar y presentar contenidos de noticias de calidad, (2) involucrar al público en un discurso informativo interactivo, (3) emplear nuevos métodos de reportes optimizados para la era digital y en red, y (4) en elaborar estrategias organizativas para un entorno digital, en red y móvil ( $p$. 183). 


\section{Metodología}

Para esta investigación se utilizará una metodología mixta utilizando como enfoque primordial tanto a lo cualitativo como lo cuantitativo dado que "todos los datos cuantitativos se basan en juicios cualitativos y cualquier dato cualitativo puede describirse y manipularse matemáticamente" (Páramo, 2011, p. 45). Por otra parte, esta metodología pretende responder a las preguntas formuladas para esta investigación, ya que "el enfoque mixto puede utilizar los dos enfoques para responder distintas preguntas de investigación de un planteamiento del problema" (Hernández Sampieri, Fernández Collado, \& Baptista Lucio, 2007, p. 755).

En el trabajo con la comunidad elegida utilizaremos lo cuantitativo para la medición de la producción de contenidos del medio, el alcance en redes sociales, las interacciones con el contenido publicado, la frecuencia de publicaciones, etc. Lo cualitativo servirá, en cambio, para evaluar el comportamiento de la comunidad de periodistas y colaboradores de La Barra Espaciadora.

Para lo anterior, se analizará el número de contenidos publicados por La Barra Espaciadora desde el 1 de diciembre de 2017 hasta el 31 de agosto de 2018 para conocer en qué momentos el medio tiene mayor producción. A la vez, se verificará durante el mismo periodo, las variaciones en cuanto al alcance en redes sociales, utilizando los datos de Facebook en relación con los contenidos publicados. En Facebook se analizaron 479 publicaciones realizadas dentro del periodo mencionado y en la web de LBE se tomó en cuenta los 97 artículos publicados durante el mismo lapso.

Asimismo, se realizará la triangulación de datos, esta será de dos tipos: de distinta naturaleza y de distintas fuentes. El primero incluye datos cuantitativos y cualitativos, mientras que el segundo, fuentes como archivos, cuestionarios, observación, etc.

El propósito del uso de la metodología es explicativo y complementario. Se busca explicar cómo funciona la sala de redacción digital y cómo el funcionamiento de esta se refleja en la cantidad, frecuencia de producción y difusión de contenidos.

Se utilizará también a la entrevista como técnica de investigación. Se conversará con el equipo base del medio que está conformado por tres personas, además de dialogar con nueve colaboradores del medio de comunicación. Solo el equipo base del medio no sufre cambios en cuanto al número de integrantes, sin embargo, sus colaboradores no siempre son los mismos. Durante el periodo de investigación el número de personas que colaboran con el medio, sin contar al equipo base, es de 20. Se realizó un total de 11 entrevistas. Las estadísticas por obtener son de tipo descriptivas, es decir, que permitirán un acercamiento a cómo funciona esta sala de redacción en específico, no se infiere sobre el comportamiento general de otras salas de redacción. 


\section{Hallazgos}

La Barra Espaciadora, como se pudo observar en la investigación, se estructura a través de círculos concéntricos, conformados por: fundadores, equipo base, colaboradores frecuentes y esporádicos, además de los lectores, dado que, de estos, surgen potenciales colaboradores de LBE.

\section{Ilustración 1. Estructura del equipo de LBE}

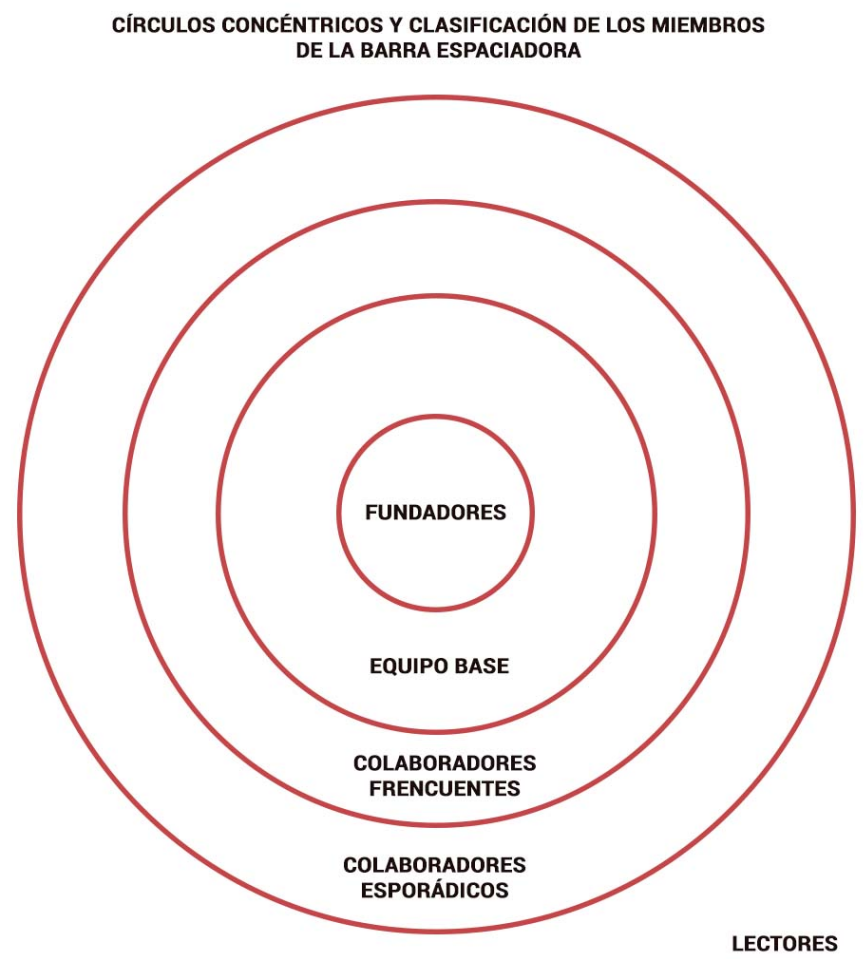

\section{Fuente: Elaboración propia}

De las entrevistas realizadas, se pudo evidenciar en cuanto a datos demográficos que, 10 de 11 entrevistados son ecuatorianos, el uno restante es español. La edad promedio de las personas que pertenecen al medio es de 35 años, con edades comprendidas entre 18 y 50 años. De ellos, 5 son hombres y 6 son mujeres.

Respecto al nivel formativo de sus miembros, de 11 entrevistados, seis poseen un título universitario relacionado con Comunicación, ya sea licenciatura en comunicación o periodismo. De estos seis, dos tienen maestría, una persona cuenta con una maestría y un doctorado y, uno de ellos, cuenta con una tecnología en radio. Dos de los entrevistados están en proceso de obtención de su título de tercer nivel en Comunicación. Tan solo tres no tienen títulos de tercer relacionados con Comunicación. Asimismo, otros tres cuentan con educación no relacionada con comunicación.

Sobre sus condiciones laborales fuera del medio, nueve personas cuentan con una actividad a tiempo completo, ya sea en el sector público o privado. Una posee un trabajo a tiempo parcial y, la persona restante trabaja solo como freelance. Asimismo, nueve de los entrevistados consideran que su colaboración es esporádica, mientras dos la 
consideran a tiempo completo. Del equipo base, solo una persona considera que se dedica a tiempo completo al medio.

Tabla 1. Descripción de la comunidad de colaboradores LBE

\begin{tabular}{|c|c|c|c|c|c|c|c|c|c|c|}
\hline Edad & Sexo & Nacionalidad & $\begin{array}{l}\text { Licenciatura } \\
\text { en } \\
\text { comunicación }\end{array}$ & $\begin{array}{c}\text { Tercer } \\
\text { nivel en } \\
\text { otras áreas }\end{array}$ & $\begin{array}{l}\text { Otra educación } \\
\text { en } \\
\text { comunicación }\end{array}$ & $\begin{array}{c}\text { Educación no } \\
\text { relacionada } \\
\text { con la } \\
\text { comunicación }\end{array}$ & $\begin{array}{l}\text { Actividad } \\
\text { laboral }\end{array}$ & $\begin{array}{c}\text { Cargo en la } \\
\text { revista }\end{array}$ & $\begin{array}{l}\text { Dedicación } \\
\text { esporádica }\end{array}$ & $\begin{array}{l}\text { Dedicación } \\
\text { a tiempo } \\
\text { completo }\end{array}$ \\
\hline 50 & Hombre & Ecuatoriana & NO & $\begin{array}{l}\text { Ingenieria } \\
\text { informática }\end{array}$ & & No & $\begin{array}{l}\text { Empleado } \\
\text { público. } \\
\text { Departamento } \\
\text { de } \\
\text { comunicación } \\
\end{array}$ & Webmaster & $x$ & \\
\hline 35 & Mujer & Ecuatoriana & sí & No & & $\begin{array}{l}\text { Administración } \\
\text { de políticas } \\
\text { públicas }\end{array}$ & $\begin{array}{l}\text { Empleada } \\
\text { pública / } \\
\text { Freelance }\end{array}$ & Cronista & $x$ & \\
\hline 41 & Hombre & Española & sí & No & $\begin{array}{l}\text { Técnico de } \\
\text { radio }\end{array}$ & $\begin{array}{l}\text { Educador } \\
\text { canino }\end{array}$ & Fotografía & Fotógrafo & $x$ & \\
\hline 18 & Mujer & Ecuatoriana & En proceso & No & & No & Estudiante & $\begin{array}{l}\text { Equipo } \\
\text { Multimedia }\end{array}$ & $x$ & \\
\hline 21 & Mujer & Ecuatoriana & Sí & No & & No & $\begin{array}{l}\text { Asistente de } \\
\text { investigación } \\
\text { universitaria }\end{array}$ & $\begin{array}{l}\text { Colaboradora } \\
\text { contenido } \\
\text { multimedia }\end{array}$ & $\mathrm{x}$ & \\
\hline 41 & Hombre & Ecuatoriana & Sí & No & $\begin{array}{l}\text { Máster en } \\
\text { antropología } \\
\text { visual }\end{array}$ & Música & $\begin{array}{l}\text { Docencia } \\
\text { universitaria/ } \\
\text { periodista/ } \\
\text { músico }\end{array}$ & $\begin{array}{l}\text { Editor } \\
\text { general }\end{array}$ & & $x$ \\
\hline 28 & Hombre & Ecuatoriana & Sí & No & $\begin{array}{l}\text { Máster en } \\
\text { comunicación } \\
\text { política }\end{array}$ & No & $\begin{array}{l}\text { Comunicador } \\
\text { en una } \\
\text { organización } \\
\text { no } \\
\text { gubernamental }\end{array}$ & $\begin{array}{l}\text { Articulista- } \\
\text { Asesor }\end{array}$ & $x$ & \\
\hline 20 & Mujer & Ecuatoriana & En proceso & No & & No & Estudiante & $\begin{array}{l}\text { Community } \\
\text { manager }\end{array}$ & $x$ & \\
\hline 50 & Hombre & Ecuatoriana & No & No & No & No & $\begin{array}{l}\text { Gestor } \\
\text { cultural/ } \\
\text { artista } \\
\text { plástico/ } \\
\text { tatuador/ } \\
\text { director de } \\
\text { arte }\end{array}$ & Ilustrador & $\mathrm{x}$ & \\
\hline 43 & Hombre & Ecuatoriana & No & $\begin{array}{l}\text { Marketing } \\
\text { y } \\
\text { publicidad }\end{array}$ & No & No & Packaging & $\begin{array}{l}\text { Director } \\
\text { comercial }\end{array}$ & & $x$ \\
\hline 41 & Mujer & Ecuatoriana & Sí & No & $\begin{array}{l}\text { Máster en } \\
\text { Estudios de la } \\
\text { Culuta/ } \\
\text { Doctorado en } \\
\text { literatura } \\
\text { latinoamericana }\end{array}$ & No & $\begin{array}{l}\text { Docencia } \\
\text { universitaria }\end{array}$ & $\begin{array}{l}\text { Colaboradora } \\
\text { artículista }\end{array}$ & $x$ & \\
\hline
\end{tabular}

Fuente: Elaboración propia

En cuanto al funcionamento de la sala de redacción digital de LBE, los entrevistados, en su mayoría, 9 de 11, la describen como una sala de redacción centralizada, dado que la interacción principal se genera únicamente con el editor. Una 
colaboradora mencionó que durante los años que lleva en LBE, solo se ha comunicado con el editor. Dos entrevistados manifestaron que la sala de redacción es multidisciplinaria, en referencia a los encargos que tienen los miembros del equipo cuando se trabaja un especial periodístico o un evento relacionado con el medio. Palabras como colaborativa, abierta, organizada y desorganizada, también surgieron en las declaraciones. Tan solo una persona la describió como descentralizada.

Tabla 2. Percepción de la estructura de la sala de redacción- LBE

\begin{tabular}{|r|r|r|r|}
\hline \multicolumn{2}{|c|}{ Pregunta 1: ¿Cómo describiría la estructura de la sala de redacción de La Barra Espaciadora? } \\
\hline Centralizada & \multicolumn{1}{|c|}{ Descentralizada } & Multidisciplinaria & Otros \\
\hline 9 & 1 & 2 & 2 \\
\hline
\end{tabular}

Fuente: Elaboración propia

Por otra parte, los miembros de LBE consideran que este medio es digital, principalmente por estar en internet. No obstante, al repreguntar en la entrevista, se pudo obtener información más detallada sobre por qué este medio podría ser digital, según la percepción de los entrevistados. La mayoría mencionó que LBE presenta un contenido editorial distinto al de los medios tradicionales, debido a que aborda temas que no necesariamente están ligados a la coyuntura nacional. También, algunos se refirieron al contenido multimedia e interactivo, pero aclararon que solo en algunos artículos se puede apreciar este tipo de elementos. Solo una persona considera que LBE no es un medio digital.

Tabla 3. Características de LBE como medio digital

\begin{tabular}{|l|l|l|l|l|}
\hline \multicolumn{7}{|c|}{ Pregunta 4: ¿Cuáles son las características que hacen que LBE sea un medio digital? } \\
\hline & & & & No es un medio \\
Multimedialidad & Hipertextualidad & Interactividad & Contenido editorial & digital \\
\hline & 2 & 6 & 10 & 1 \\
\hline
\end{tabular}

Fuente: Elaboración propia

Las respuestas de las entrevistas dejan en manifiesto que, a la hora de referirnos a comunicación, interacciones y trabajo colectivo, las personas que forman parte del equipo de LBE, en su gran mayoría, solo tienen contacto con el editor general, 6 de 11, y solo conocen por redes sociales digitales a los demás colaboradores, 6 de 11, pese a que algunos mencionaron que, a ciertos miembros los conocen en redes sociales y a otros personalmente. Inclusive el editor general indicó que existen colaboradores a los que únicamente conoce por internet.

En el caso de las personas que dicen tener otra interacción con algunos miembros de LBE, 5 de 11, coinciden en que, dichas interacciones, se producen con una persona del equipo base, ya sea el webmaster o la editora multimedia.

Al preguntar sobre los factores que afectan al desarrollo de un periodismo de mejor calidad en la rutina laboral de LBE, diez de los entrevistados indicaron que el factor económico/financiero, es una gran limitante, especialmente a la hora de enviar a los 
periodistas a realizar investigaciones de campo. De igual forma, diez entrevistados indicaron que otra limitante es la condicionante laboral/ salarial, para ellos, es esencial que exista un salario o pago por publicación para que la itinerancia en la sala de redacción sea menor y para que el compromiso de quienes son parte del medio sea mayor. Para ocho entrevistados, una condicionante, también es la condición del trabajo, argumentan que, al no tener un contrato o un escrito que defina funciones y tiempos de publicación, el compromiso no es real. Otras condicionantes, que también surgieron entre la opinión de los entrevistados, fueron: condicionante de tipo formativo, tecnológico e ideológico, rutinas periodísticas y, se mencionó, a la "fuga de talentos" y la falta de un espacio físico.

En cuanto a la condicionante formativa, las respuestas indicaron que no todos los miembros provienen de la misma área ni tienen los mismos conocimientos teóricos o técnicos, por ejemplo, no todos conocen cómo manejar nuevos lenguajes en internet o mínimos básicos de programación. Quien mencionó como condicionante a las rutinas periodísticas indicó que, con el advenimiento de internet, los periodistas deben conocer sobre reportería digital y cómo trabajar tanto en el ambiente tradicional y digital, de ahí que existe un cambio dentro de estas rutinas.

La condicionante tecnológica, en cambio, fue considerada como una limitante a la hora de acceder a los contenidos que crea el medio, ya que, sin las herramientas tecnológicas necesarias, los plugins, espacio en servidores, mantenimiento del sitio web o licencias de funcionamiento de ciertas aplicaciones, el sitio carga más lento o los contenidos no tienen la calidad esperada. Esta condicionante está ligada al aspecto económico, dado que varios elementos tecnológicos requieren una inversión económica.

Sobre la condicionante de tipo ideológico, esta fue entendida para los entrevistados como la ideología del medio, quien se refirió a ella, señaló que es importante que LBE tenga un estatuto que de las pautas sobre los contenidos y líneas que sigue. Sin eso, considera esta persona, se trabaja a partir de la ambigüedad.

Tabla 4. Condicionantes que afectan a las rutinas laborales de LBE

\begin{tabular}{|} 
Pregunta 5: ¿Cuáles son las condicionantes que afectan al desarrollo de un periodismo de mejor \\
calidad en su rutina laboral de LBE? \\
Ideológicos
\end{tabular}

Fuente: Elaboración propia

Se debe agregar que, de los 11 entrevistados, 10 de ellos quisieran trabajar exclusivamente y a tiempo completo en el medio, siempre y cuando, se reciba un salario. A uno de los entrevistados no le interesa el trabajo a tiempo completo de ningún tipo. 


\section{Discusión y conclusiones}

Como conclusiones preliminares, podemos sugerir que Rheingold (2007) no se equivocaba al decir que:

Las multitudes inteligentes consisten en personas que actúan concertadamente inclusive si no se conocen. Las personas que forman parte de estas multitudes cooperan entre sí de formas que antes no eran posibles porque ahora llevan dispositivos que poseen ambas: la comunicación y las capacidades computacionales (p. 12).

No obstante, que la interacción dentro de la sala de redacción sea, principal y casi exclusivamente con una persona, es alarmante. Si bien los miembros se conocen de alguna forma y colaboran cuando se debe realizar un especial periodístico, el trabajo diario se concentra en la relación editor- periodista y, en pocos casos, se suma a una tercera persona. No existen reuniones semanales para discutir los contenidos a publicar, estos surgen por iniciativa de los colaboradores y, en ocasiones especiales, a petición del editor.

Los miembros de LBE se conocen por redes sociales y su interacción se limita en compartir contenido y comentar, en algunos casos, las publicaciones o artículos de sus otros colegas. Las redes sociales digitales, entonces, permiten a los periodistas "mantenerse en contacto con sus colegas y su red profesional" (Paulussen \& Harder, 2014 , p. 2). Así, "encuentran nuevos contactos, y discuten noticias y problemas" (Paulussen \& Harder, 2014, p. 2) de manera similar a como se lo solía hacer en salas de redacción. Por su parte, Hermida manifiesta que las redes sociales ayudan al periodista "monitorear la constante transmisión de noticias e información y detectar tendencias y cuestiones bajo el radar de noticias" (2010, p. 302). Considerando la propuesta de autores como Rheingold, Paulussen, Harder y Hermida, se puede sugerir que, para el correcto funcionamiento de la sala de redacción digital, basta con que los miembros se conozcan a través de redes sociales digitales, no obstente es necesario que su interacción vaya más allá del "me gusta" o el "compartido". Las reuniones de tipo editorial y la construcción de los contenidos periodísticos deben involucrar a los miembros del equipo siempre, no solo en caso de especiales periodísticos.

Pese a que los entrevistados no consideren como una gran limitante a las rutinas periodísticas, se debe tener presente que las rutinas periodísticas, se convierten en el punto de inflexión necesario para la comparación de las características de la labor periodística que se ha desarrollado en América Latina (Gutiérrez-Atala, Odriozola-Chéné, Ferreira, Anaya-Ávila, \& Pajoni, 2015, p. 88).

La inmediatez, cuyo valor se ha intensificado para el periodismo debido a la irrupción de los cibermedios, desemboca en el desarrollo de nuevos procesos periodísticos y en la disminución del control ejercido por el periodista en la narración de los acontecimientos (Odriozola-Chéné, Aguirre-Mayorga, \& Roa-Chejín, 2016, p. 94). De igual forma, un miembro de un medio digital debe: "(1) crear, entregar y presentar contenidos de noticias de calidad, (2) involucrar al público en un discurso informativo interactivo, (3) emplear nuevos métodos de reportes optimizados para la era digital y en red, y (4) en elaborar estrategias organizativas para un entorno digital, en red y móvil" 
(Pavlik, 2013, p. 183), y son, justamente estas funciones las que no quedan claras dentro de los miembros de LBE, como se pudo evidenciar al momento de preguntar por qué este medio podría ser considerado como digital. Si los periodistas y colaboradores no tienen claro que, para ser un medio digital no solo basta con estar en internet, difícilmente se puede brindar un contenido adaptado a los lenguajes que los usuarios en la web demandan.

Si bien, autores como Pavlik (2013) indican que algunos medios de comunicación nativos digitales notaron que "mediante la creación y gestión de una sala de redacción virtual, la gestión de los medios de comunicación puede reducir drásticamente el coste reduciendo o incluso eliminando la sala de noticias central y física” ( p. 187), la reducción de costos y a la posibilidad de no necesitar un espacio físico para distribuir el trabajo, las tecnologías digitales trajeron otros beneficios: "un enriquecimiento en los lenguajes informativos, en las modalidades de relación con el público, en la ubicuidad y temporalidad de la información y, en fin, en múltiples ámbitos cruciales de la actividad periodística" (Salaverría, 2009, p. 2). Se puede concluir que, en el caso analizado, la condicionante económica y salarial es, para los colaboradores y periodistas, el principal problema para el funcionamento del medio, esto considerando que si bien existe un abaratamiento en cuanto a costos y, por parte de los lectores, la colaboración con textos, etc. Al no existir un pago o un contrato, el nivel de compromiso es mucho menor, provocando que la estabilidad dentro de la sala de redacción sea mínima.

Con los datos recolectados, se puede describir a la sala de redacción de LBE como un sala de redacción digital centralizada, donde, si bien existe colaboración en circunstancias especiales, no hay trabajo concertado entre los distintos miembros del equipo, como el webmaster, la editora multimedia, los periodistas, los fotógrafos y los encargados de la difusión de contenidos.

Para responder a la pregunta de investigación: ¿Cómo debe funcionar la comunidad virtual de colaboradores y periodistas, sus rutinas periodísticas y el trabajo en la sala de redacción de un medio digital - LBE- para la creación y difusión de contenidos?, en este primer momento, se puede sugerir que la estructura debe pasar de centralizada a interdisciplinaria, donde la delegación de funciones permita que exista mayor participación y colaboración en el trabajo diario. También, es de vital importancia que no exista la precarización de la labor periodística, el salario, el pago por publicación y el costeo de viáticos u otros gastos a la hora de realizar coberturas es necesario para la creación de contenidos y el cumplimiento de los plazos de entrega. Además, se sugiere trabajar sobre las rutinas periodísticas y las condicionantes formativas, para que así, los periodistas y colaboradores estén conscientes de que ser un medio digital va más allá del hecho de estar en internet. 


\section{Bibliografía}

Canter, L. (2015). Personalised tweeting: The emerging practices of journalists on Twitter. Digital Journalism, 3(6), 888-907.

Fernández Serrano, E. (2014). Sociedad en Red Manuel Castell [Mp4]. Recuperado de https://www.youtube.com/watch?v=qpkENiSUcJM

Gutiérrez-Atala, F., Odriozola-Chéné, J., Ferreira, J., Anaya-Ávila, P., \& Pajoni, H. (2015). El peso de la presión social y política: estudio de las limitaciones que condicionan el desempeño de los periodistas en cinco países latinoamericanos. Anuario electrónico de estudios en Comunicación Social “ Disertaciones”, 9(1), 84-102.

Hermida, A. (2010). Twittering the news: The emergence of ambient journalism. Journalism practice, 4(3), 297-308.

Hernández Sampieri, R., Fernández Collado, C., \& Baptista Lucio, P. (2007). Metodología de la investigación (4ta ed.). México: McGraw-Hill.

Jenkins, H. (2015). Fans, blogueros y videojuegos: la cultura de la colaboración. Barcelona: Paidós.

Lewis, S. C., Holton, A. E., \& Coddington, M. (2014). Reciprocal Journalism. Journalism Practice, 8(2), 229-241. https://doi.org/10.1080/17512786.2013.859840

Llorca-Abad, G., \& Cano-Orón, L. (2016). Espacio y tiempo en el siglo XXI: velocidad, instantaneidad y su repercusión en la comunicación humana. Revista ComHumanitas, 6(1), 219-233.

Odriozola-Chéné, J., Aguirre-Mayorga, C., \& Roa-Chejín, M. S. (2016). Los condicionantes en el desarrollo de una buena praxis periodística en Ecuador: estudio de los factores de influencia según los profesionales ecuatorianos. ComHumanitas: revista científica de comunicación, 7(1), 89-109.

Páramo, P. (Ed.). (2011). La investigación en ciencias sociales: estrategias de investigación (1. ed). Bogotá: Universidad Piloto de Colombia.

Paulussen, S., \& Harder, R. A. (2014). Social media references in newspapers: Facebook, Twitter and YouTube as sources in newspaper journalism. Journalism Practice, $8(5), 542-551$.

Pavlik, J. V. (2013). Innovation and the future of journalism. Digital Journalism, 1(2), 181-193. https://doi.org/10.1080/21670811.2012.756666

Recuero, R., Amaral, A., \& Monteiro, C. (2012). Fandoms, trending topics and social capital in Twitter. Selected Papers of Internet Research, 2, 1-24.

Rheingold, H. (2007). Smart mobs: The next social revolution. Basic books. 
Salaverría, R. (2009). Los medios de comunicación ante la convergencia digital. En Actas del I Congreso Internacional de Ciberperiodismo y Web 2.0 (pp. 11-23). Bilbao: Servicio Editorial de la Universidad del País Vasco. Recuperado de http://dadun.unav.edu/handle/10171/5099 